\title{
Effects of Humic Acid and Chitosan under Different Levels of Nitrogen and Potassium fertilizers on Growth and Yield potential of Potato plants (Solanum tuberosum, L.)
}

\author{
Harfoush, E. A., A. H. Abdel-Razzek, F. I. El-Adgham and A. M. El-Sharkawy \\ Department of Vegetable Crops, Fac. Agric., Alexandria Univ., Alex. Egypt
}

Received on: 4/1/2017

Accepted: $28 / 2 / 2017$

\begin{abstract}
Two field experiments were carried out during the fall seasons of 2014 and 2015 to investigate the main effect of humic acid ( 0 and $1000 \mathrm{ppm})$, chitosan $(0$ and $250 \mathrm{ppm})$ and NK $(0,25,50,75$, and 100\%) of the recommended rate for the commercial production of potato as well as their various interactions on some vegetative growth characters, tubers yield and its components. The obtained results indicated that increasing the applied level of NK up to $100 \%$ constantly increased most of vegetative growth characters (foliage fresh weight, leaf area, plant height, number of main stems and leaf chlorophyll content) and tubers yield and its components (total tuber yield plant ${ }^{-1}$ and fed $^{-1}$ and tubers yield of large, medium and small sized plant $\left.{ }^{-1}\right)$. The highest two NK levels (75 and $\left.100 \%\right)$ didn't, significantly, differ in their effects on most of vegetative traits. Application of humic acid or chitosan produced, significantly, increased growth parameters as well as tubers yield and its components. The most favorable combination treatments that produced the highest mean values of growth and tubers yield as well as yield components were 75 or $100 \% \mathrm{NK}+$ humic acid or chitosan, humic acid + chitosan and 75 or $100 \% \mathrm{NK}+$ humic acid + chitosan. Therefore, fertilizing potato plants with 75 or $100 \%$ of the recommended dose of NK combined with foliar spray of humic acid and chitosan could be recommended to gain the best results.
\end{abstract}

Keywords: Humic acid, Chitosan, Nitrogen, Potassium, Potato and growth promoting.

\section{INTRODUCTION}

The potato (Solanum tuberosum, L.) is a starchy tuberous crop related to family solanaceae. It is considered the most important vegetable crops in the world. In Egypt, the potato crop ranks the fourth in economic importance after wheat, maize and rice. It is, also, the first alternative for grain crops and is used for human consumption and animal feed and as a source of starch, carbohydrates, alcohol and protein. In term of nutrition, potato is known for its higher carbohydrate content, a good source of potassium which is important for a healthy blood pressure and contribute a significant amount of vitamin $\mathrm{C}$ which is a powerful antioxidant and important for immune health as well. Egypt, among the top potato exporters globally and ranked the first in Africa. Nutrition either by mineral and/or organic fertilizers is considered the most important agricultural practice which affects the duration of growing period, plant foliage, tubers formation and quality. The necessity of nitrogen $(\mathrm{N})$ and potassium (K) for growth has been demonstrated by several investigators. Nitrogen supply was desirable for vegetative growth, dry matter accumulation and nutrients uptake by potato plants. Potassium is a mobile element in plant tissue and plays an important role in photosynthesis through carbohydrate metabolism, osmotic regulation, nitrogen uptake and translocation of assimilates. It, also, has a role in physiological processes in plant respiration, transpiration, translocation of sugars and carbohydrates and enzyme transformation. Nitrogen and potassium as macronutrients are commonly applied to the soil. Adequate NK percentage leads to maximum tubers yield and quality of potato. In addition, it possible to demonstrate characteristic differences between effect of $\mathrm{N}$ - fertilization and varied $\mathrm{N}$ - concentrations on tuber yield and composition. Humic acid is known to be among the most bio-chemically active materials found in the soil. It's an affective agent to be used as a complement to synthetic or organic fertilizers. In many instances, regular humic acid presence will reduce the need of fertilization due to the soil's and plant's ability to make better use of it. In some occurrences, fertilization can be eliminated entirely if sufficient organic materials are present which lead to a self-sustaining soil as a result of microbial processes and humus production. In general, increasing certain levels of humic acid have a number of potential benefits for plants; the most important ones are increasing water and nutrients holding capacity, enhancing solubility of phosphorus and increasing potato tubers yield and quality (Selim et al., 2009). Addition of humic substances with NPK fertilizers resulted in a lesser leaching of $\mathrm{N}$ and $\mathrm{K}$ to a deeper soil layer and higher available $\mathrm{P}$ leading to enhancement of tubers yield, quality and nutritional status of potato organs. On the other hand, Ahmed (2012) found that applied humic substances to the soil can increase irrigation and nutrition efficiency and plays a considerable 
role in increasing plant resistance against common potato diseases. Chitosan belongs to the carbohydrate family which contains ungratified chains formula. Chitosan is originally formulated from the glucose circle and contains a group of free amino on carbon atom number 2 (called glucose amino) which is similar to cellulous. It is obtained by alkaline deacetylation of chitin extracted from exoskeleton of crustaceans such as shrimps and crabs, as well as, from the cell walls of some fungi (Badawy et al., 2011). Researches indicated that chitosan can be used to enhance plants' growth and fruit yield and has been used in several applications in the agricultural field (Sheikha et al., 2011). Chitosan, also, enhances the plant defense and is used for controlling potato diseases and improving yield and quality (Halina, 2008, a, b).

Therefore, the scope of the present study is to identify the main and interaction effects of foliar application of humic acid and chitosan under different of NK levels on morphological traits and tubers yield and its components.

\section{MATERIALS AND METHODS}

Two field experiments were carried out during the fall seasons of 2014 and 2015 at El-Rowysat, ElHamam district, Matrouh Governorate, Egypt. These experiments aimed to investigate the effect of different treatments of humic acid and chitosan under various levels of nitrogen and potassium on vegetative growth and tubers yield and its components of potato cv. Agila.

Preceding the initiation of each experiment, soil samples at $25 \mathrm{~cm}$ depth were collected and analyzed for some physical and chemical properties of the experimental site according to the published procedures described by Black (1965). The results of these analyses are listed in Table 1.

Five levels of mineral NK fertilizer of the recommended rate for commercial production of potato $(0,25,50,75$ and $100 \%)$ were used. The recommended of $\mathrm{N}$ and $\mathrm{K}_{2} \mathrm{O}$ for potato production were 150 and $75 \mathrm{Kg} \mathrm{fed}^{-1}$, orderly. The respective $\mathrm{N}$ and $\mathrm{K}$ sources were ammonium nitrate $(33.5 \%$ $\mathrm{N})$ and potassium sulfate $\left(50 \% \mathrm{~K}_{2} \mathrm{O}\right)$. The nitrogen fertilizer was applied at three equal portions starting from $5^{\text {th }}$ weeks after planting and at two weeks interval. Potassium fertilizer was side banded at two equal applications at the beginning of tuber formation and again at two weeks later.

Humic acid at two concentrations ( 0 and 1000 ppm) was used which were applied at four times; the first one was done as a soil application after two weeks of planting and the three reminder applications were foliar sprayed starting after six weeks from planting at two weeks interval. Pure chitosan at two concentrations (0 and $250 \mathrm{ppm}$ ) was used which were foliar applied at four times starting from seven weeks after planting then at two weeks interval. Each experiment included twenty combination treatments in total.

The experimental layout was a split-split plots based on Randomized Complete Blocks design with three replications. The five levels of NK fertilization were randomly arranged in the main plots and two humic acid concentrations were randomly occupied in the sub-plots while, the two chitosan concentrations were randomly allocated in the sub-sub plots. Each sub-sub plot consisted of three rows, $4.0 \mathrm{~m}$ length and $0.70 \mathrm{~m}$ width having an area $8.4 \mathrm{~m}^{2}$. Seed tubers were sown on August $15^{\text {th }}$ in 2014 and 2015 season at inrow spacing of $30 \mathrm{~cm}$. A guard row was left between each two adjusted sub-sub plots to protect against side effect. All experimental units received identical levels of phosphorus (75kg P2O5 $\left.\mathrm{Fed}^{-1}\right)$ and farmyard manure $\left(20 \mathrm{~m}^{3} \mathrm{fed}^{-1}\right)$ during the preparation of experimental site. The first row was saved for vegetative growth characters while, the $2^{\text {nd }}$ and $3^{\text {rd }}$ rows were allocated for tubers yield and its components. The harvested plot area was $5.6 \mathrm{~m}^{2}$. All agricultural practices such as irrigation, cultivation, diseases and pests control were carried out whenever it was necessary according to the commercial production of potato.

\section{Data Recorded}

\section{Vegetative growth characters}

Five random plants from each plot were taken after 70 days of planting and the following data were recorded; plant height $(\mathrm{cm})$, number of leaves and main stems, foliage fresh weight (gm), Leaf area $\left(\mathrm{cm}^{2}\right)$ and leaf chlorophyll content plant ${ }^{-1}$ (mg/100g FW)

Table 1: Soil's physical and chemical properties of the experimental site through the two growing seasons of 2014 and 2015.

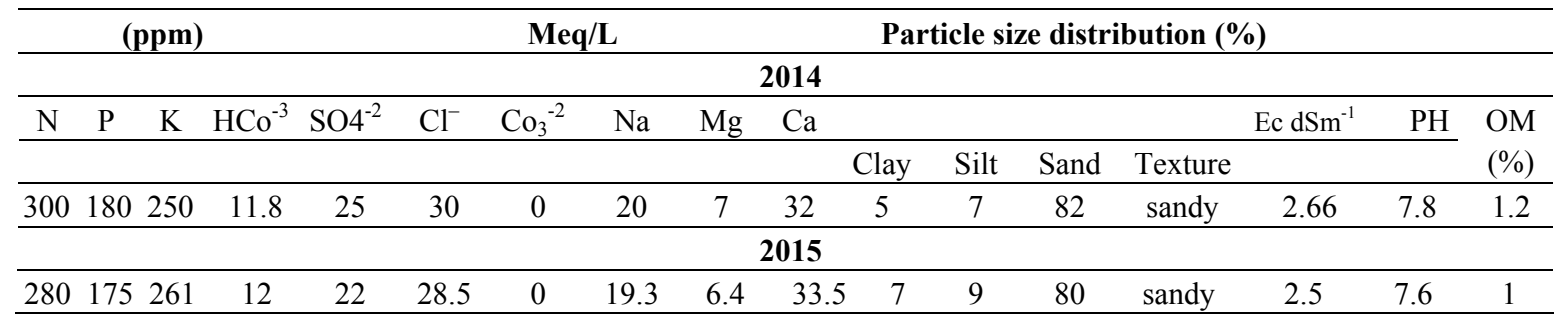

*These analyses were carried out at the Central Laboratory, Faculty of Agriculture, Alexandria University. 


\section{Tubers yield and its components}

At harvest time, the following measurements was recorded; total tubers yield; all harvested tubers from each sub-sub plot were weighed and converted into tones $\mathrm{fed}^{-1}$, average tubers yield plant ${ }^{-1}$; calculated as average tubers weight of ten random plants from each experimental unite and tubers size (grading) plant ${ }^{-1}$; all harvested tubers plant $^{-1}$ was sorted into three sizes; large tubers $(>60 \mathrm{~mm}$ in dimeter), medium tubers (46-60 $\mathrm{mm}$ in dimeter) and small tubers ( $<46 \mathrm{~mm}$ in diameter). Each size was weighted.

\section{Statistical analysis}

Appropriate analysis of variance, on obtained data, according to the statistical design was performed. New Least Significant Difference (AlRawi and Kalf- Allah, 1980) was utilized to verify difference between treatment means.

\section{RESULTS AND DISCUSSION Vegetative growth characters}

The results shown in Tables 2-6 illustrated the main and different order interactions of NK fertilizer, humic acid and chitosan on the studied vegetative growth characters of potato plants, in the two growing seasons.

The results in Table 2 indicated, generally, that increasing applied NK level, up to $100 \%$ of the recommended rate, progressively and significantly, increased canopy fresh weight, leaf area and leaf chlorophyll content plant ${ }^{-1}$. The highest two NK levels (75 and $100 \%$ ) didn't, significantly, differ in their effect on foliage fresh weight plant $^{-1}$. Meanwhile, number of main stems and plant height didn't, significantly, respond to NK levels, in the two seasons. The highest number of leaves plant ${ }^{-1}$ was recorded on plants fertilized with NK at $100 \%$ level in the $2^{\text {nd }}$ season. The results revealed, also, that treated-humic acid plants, significantly, increased mean values of all studied vegetative growth parameters as compared with untreatedhumic acid plants (control). Moreover, foliar spray with chitosan, significantly, increased number of leaves, canopy fresh weight, leaves area and leaf chlorophyll content plant ${ }^{-1}$ meanwhile, number of stems and stature plant ${ }^{-1}$ didn't, significantly, respond to chitosan treatments.

Table 3 and 4 showed the influence of $1^{\text {st }}$ order interactions between any two studied factors on vegetative growth features, in the fall seasons of 2014 and 2015. Regarding the interaction effects between NK levels and humic acid concentrations, the results showed that, increasing the levels of applied NK to treated-humic acid plants, significantly, increased the studied growth characters, but with different magnitudes. The greatest mean values of all studied characters were, generally, recorded from plants fertilized with 75 or $100 \%$ NK levels combined with foliar application of humic acid at $1000 \mathrm{ppm}$. Concerning the interaction effects between NK levels and chitosan concentrations, the best treatment combination that produced the highest, significant, mean values in plant height, foliage fresh weight, leaf area, number of leaves and main stems and leaf chlorophyll content plant $^{-1}$ appeared to be those involved NK fertilization rate of 75 or $100 \%$ of the recommended dose together with the foliar application of chitosan at $250 \mathrm{ppm}$. Regarding the interaction between humic acid and chitosan concentrations, results revealed, generally, that the potato treated plants with humic acid and chitosan at 1000 and $250 \mathrm{ppm}$, orderly produced the highest mean values of all studied vegetative growth characters. However, foliar application of chitosan at 0 and $250 \mathrm{ppm}$ together with humic at $1000 \mathrm{ppm}$ didn't, significantly, differ in their effects on number of main stems and leaves plant ${ }^{-1}$, during 2015 season .

The results concerning the effect of the $2^{\text {nd }}$ order interaction among the three studied factors on vegetative growth characters were shown in Tables 5-6. Comparisons among the means of various combination treatments, generally, illustrated the presence of some, true, interaction effects, in both seasons. The results, generally, indicated that increasing the levels of applied NK fertilizer combined with foliar application of humic acid and chitosan, significantly and progressively, increased canopy fresh weight, leaves area and chlorophyll content plant $^{-1}$ compared to those of untreated with humic acid and chitosan treatments. The results, also, showed that the highest mean values of number of leaves and main stems as well as plant stature were recorded from the plants fertilized with $\mathrm{NK}$ at 75 or $100 \%$ of the recommended rate combined with humic acid and chitosan foliar spraying.

The stimulation effect of applying $\mathrm{N}$ on vegetative growth characters of potato plants may be attributed to the well-known functions of $\mathrm{N}$ in plant life. Being a part of protein is an important constituent of protoplasm, as well as enzymes, the biological catalytic agents, which speed life possesses, have $\mathrm{N}$ as the major constituents. Moreover, $\mathrm{N}$ involves in many organic compounds of plant system. A sufficient supply of various nitrogenous compounds is therefore, required in each plant cell for its proper functioning (Mengel and Kirkby, 1987, Anabousi, et al 1997; and Ahmad, et al 2009). Also, nitrogen fertilizer increased the leaf area which increases the amount of solar radiation intercepted and consequently, increases dry matter production of different plant parts (Krishnappa et al., 1989). These results are in close agreement with the findings of (Pervez et al., 2013, and Bourke, 1985). 


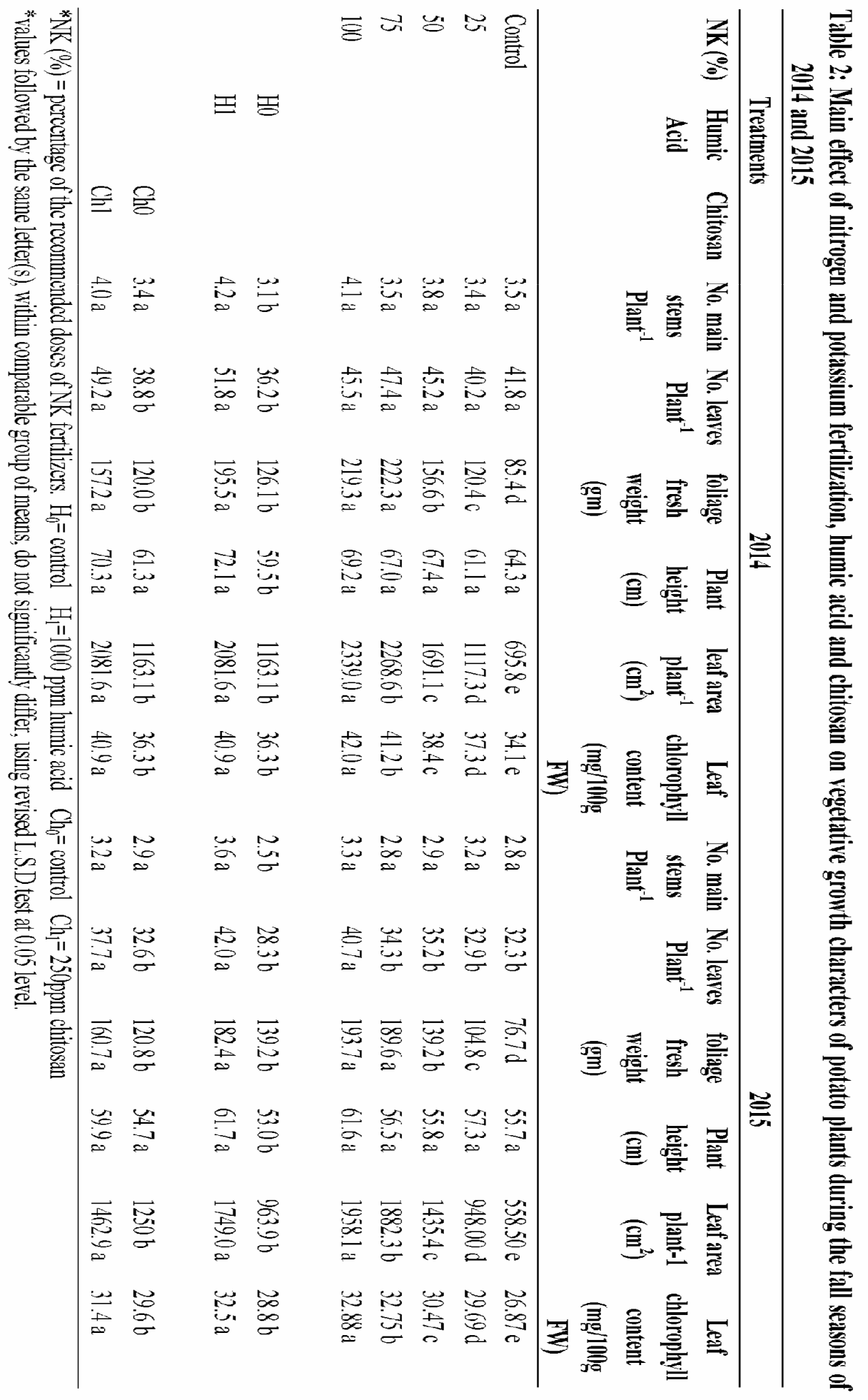


Table 3: First order interaction effect between NK fertilization, humic acid and chitosan on vegetative growth characters of potato plants during the fall seasons of 2014 and 2015

\begin{tabular}{|c|c|c|c|c|c|c|c|c|}
\hline \multicolumn{3}{|c|}{ Treatments } & \multicolumn{3}{|c|}{2014} & \multicolumn{3}{|c|}{2015} \\
\hline NK (\%) & $\begin{array}{l}\text { Humic } \\
\text { Acid }\end{array}$ & Chito-san & $\begin{array}{c}\text { No. main } \\
\text { stems } \\
\text { Plant }^{-1}\end{array}$ & $\begin{array}{c}\text { No. leaves } \\
\text { Plant }^{-1}\end{array}$ & $\begin{array}{c}\text { foliage } \\
\text { fresh } \\
\text { weight } \\
\text { (gm) }\end{array}$ & $\begin{array}{c}\text { No. main } \\
\text { stems } \\
\text { Plant }^{-1}\end{array}$ & $\begin{array}{c}\text { No. leaves } \\
\text { plant }^{-1}\end{array}$ & $\begin{array}{c}\text { foliage } \\
\text { fresh } \\
\text { weight } \\
\text { (gm) }\end{array}$ \\
\hline \multirow[t]{2}{*}{ Control } & $\mathrm{HO}$ & & $3.0 \mathrm{~b}$ & $35.7 \mathrm{~b}$ & $73.9 \mathrm{~h}$ & $2.5 \mathrm{c}-\mathrm{e}$ & 29 e-f & $68.3 \mathrm{f}$ \\
\hline & H1 & & $4.0 \mathrm{ab}$ & $47.9 \mathrm{ab}$ & $96.9 \mathrm{gh}$ & $3.2 \mathrm{bc}$ & $36 \mathrm{c}-\mathrm{d}$ & $85.0 \mathrm{ef}$ \\
\hline \multirow[t]{2}{*}{25} & $\mathrm{H} 0$ & & $3.0 \mathrm{~b}$ & $34.4 \mathrm{~b}$ & $101.3 \mathrm{fg}$ & $2.7 \mathrm{c}-\mathrm{e}$ & $29 \mathrm{e}-\mathrm{g}$ & $89.7 \mathrm{~d}-\mathrm{f}$ \\
\hline & $\mathrm{H} 1$ & & $3.8 \mathrm{ab}$ & $45.9 \mathrm{ab}$ & $139.4 \mathrm{de}$ & $3.7 \mathrm{ab}$ & $42 \mathrm{ab}$ & $119.8 \mathrm{~cd}$ \\
\hline \multirow[t]{2}{*}{50} & $\mathrm{HO}$ & & $3.2 \mathrm{~b}$ & $35.3 \mathrm{~b}$ & $126.1 \mathrm{ef}$ & $2.3 \mathrm{de}$ & $27 \mathrm{fg}$ & $117.2 \mathrm{c}-\mathrm{e}$ \\
\hline & $\mathrm{H} 1$ & & $4.4 \mathrm{ab}$ & $55.1 \mathrm{a}$ & $187.1 \mathrm{~b}$ & $3.5 \mathrm{ab}$ & 39 b-d & $161.2 \mathrm{~b}$ \\
\hline \multirow[t]{2}{*}{75} & $\mathrm{HO}$ & & $3.0 \mathrm{~b}$ & $39.4 \mathrm{~b}$ & $161.4 \mathrm{~cd}$ & $2.0 \mathrm{e}$ & $25 \mathrm{~g}$ & $137.3 \mathrm{bc}$ \\
\hline & $\mathrm{H} 1$ & & $4.0 \mathrm{ab}$ & $55.3 \mathrm{a}$ & $283.2 \mathrm{a}$ & $3.7 \mathrm{ab}$ & $44 a$ & $241.8 \mathrm{a}$ \\
\hline \multirow[t]{2}{*}{100} & $\mathrm{HO}$ & & $3.4 \mathrm{ab}$ & $36.5 \mathrm{~b}$ & $168.0 \mathrm{bc}$ & $3.0 \mathrm{~b}-\mathrm{d}$ & 33 d-f & $154.7 \mathrm{~b}$ \\
\hline & $\mathrm{H} 1$ & & $4.8 \mathrm{a}$ & $54.5 \mathrm{a}$ & $270.7 \mathrm{a}$ & $4.2 \mathrm{a}$ & $49 \mathrm{a}$ & $232.7 \mathrm{a}$ \\
\hline \multirow[t]{2}{*}{ Control } & & $\mathrm{Ch} 0$ & $3.2 \mathrm{bc}$ & $38.1 \mathrm{~cd}$ & $83.5 \mathrm{e}$ & $2.7 \mathrm{ab}$ & $30 \mathrm{a}-\mathrm{c}$ & $69.7 \mathrm{f}$ \\
\hline & & Ch1 & $3.8 \mathrm{a}-\mathrm{c}$ & $45.5 \mathrm{a}-\mathrm{c}$ & $87.2 \mathrm{e}$ & $3.0 \mathrm{ab}$ & $34 \mathrm{a}-\mathrm{c}$ & $83.7 \mathrm{f}$ \\
\hline \multirow[t]{2}{*}{25} & & Ch0 & $3.2 \mathrm{bc}$ & $35.1 \mathrm{~d}$ & $105.9 \mathrm{~d}$ & $3.2 \mathrm{ab}$ & $35 \mathrm{a}-\mathrm{c}$ & 93.7 ef \\
\hline & & Ch1 & $3.6 \mathrm{a}-\mathrm{c}$ & $45.3 \mathrm{bc}$ & $134.8 \mathrm{c}$ & $3.2 \mathrm{ab}$ & $36 \mathrm{a}-\mathrm{c}$ & $115.8 \mathrm{de}$ \\
\hline \multirow[t]{2}{*}{50} & & $\mathrm{Ch} 0$ & $3.6 \mathrm{a}-\mathrm{c}$ & $39.6 \mathrm{~cd}$ & $141.1 \mathrm{c}$ & $2.5 \mathrm{~b}$ & $28 \mathrm{c}$ & $126.2 \mathrm{~cd}$ \\
\hline & & Ch1 & $4.0 \mathrm{ab}$ & $50.8 \mathrm{ab}$ & $172.1 \mathrm{~b}$ & $3.3 \mathrm{ab}$ & $38 \mathrm{a}-\mathrm{c}$ & $152.2 \mathrm{bc}$ \\
\hline \multirow[t]{2}{*}{75} & & $\mathrm{Ch} 0$ & $3.0 \mathrm{c}$ & $40.4 \mathrm{~cd}$ & $179.8 \mathrm{~b}$ & $2.7 \mathrm{ab}$ & $30 \mathrm{a}-\mathrm{c}$ & $152.5 \mathrm{bc}$ \\
\hline & & Ch1 & $4.0 \mathrm{ab}$ & $54.3 \mathrm{a}$ & $264.9 \mathrm{a}$ & $3.0 \mathrm{ab}$ & $39 a-c$ & $226.7 \mathrm{a}$ \\
\hline \multirow[t]{6}{*}{100} & & Ch0 & $3.8 \mathrm{a}-\mathrm{c}$ & $41.0 \mathrm{~cd}$ & $185.8 b$ & $3.5 \mathrm{ab}$ & $40 \mathrm{ab}$ & $162.2 \mathrm{~b}$ \\
\hline & & Ch1 & $4.4 \mathrm{a}$ & $50.0 \mathrm{ab}$ & $252.9 \mathrm{a}$ & $3.7 \mathrm{a}$ & $41 \mathrm{a}$ & $225.2 \mathrm{a}$ \\
\hline & $\mathrm{H} 0$ & Ch0 & $2.9 \mathrm{c}$ & $32.8 \mathrm{c}$ & $122.6 \mathrm{c}$ & $2.4 \mathrm{~b}$ & $27.1 \mathrm{c}$ & $107.4 \mathrm{c}$ \\
\hline & & Ch1 & $3.3 \mathrm{bc}$ & $39.7 \mathrm{~b}$ & $129.7 \mathrm{c}$ & $2.6 \mathrm{~b}$ & $29.5 \mathrm{c}$ & $119.5 \mathrm{c}$ \\
\hline & $\mathrm{H} 1$ & $\mathrm{Ch} 0$ & $3.8 \mathrm{~b}$ & $44.9 \mathrm{~b}$ & $155.9 \mathrm{~b}$ & $3.4 \mathrm{a}$ & $38.1 \mathrm{a}$ & $134.3 \mathrm{~b}$ \\
\hline & & Ch1 & $4.7 \mathrm{a}$ & $58.7 \mathrm{a}$ & $235.0 \mathrm{a}$ & $3.9 \mathrm{a}$ & $45.9 \mathrm{a}$ & $201.9 \mathrm{a}$ \\
\hline
\end{tabular}

*NK $(\%)=$ percentage of the recommended doses of NK fertilizers. $\mathrm{H} 0=$ control $\mathrm{H} 1=1000$ ppm humic acid $\mathrm{Ch} 0=$ control $\mathrm{Ch} 1=250 \mathrm{ppm}$ chitosan

*values followed by the same letter(s), within comparable group of means, do not significantly differ, using revised L.S.D.test at 0.05 level. 
Table 4: First order interaction effect between NK fertilization, humic acid and chitosan on vegetative growth characters of potato plants during the fall seasons of 2014 and 2015

\begin{tabular}{|c|c|c|c|c|c|c|c|c|}
\hline \multicolumn{3}{|c|}{ Treatments } & \multicolumn{3}{|c|}{2014} & \multicolumn{3}{|c|}{2015} \\
\hline NK (\%) & $\underset{\text { Acid }}{\text { Humic }}$ & Chitosan & $\begin{array}{l}\text { No. main } \\
\text { stems }_{\text {Plant }^{-1}}\end{array}$ & $\begin{array}{l}\text { No. leaves } \\
\text { Plant }^{-1}\end{array}$ & $\begin{array}{c}\text { foliage } \\
\text { fresh } \\
\text { weight } \\
\text { (gm) }\end{array}$ & $\begin{array}{c}\text { No. } \\
\text { main } \\
\text { stems } \\
\text { Plant }^{-1}\end{array}$ & $\begin{array}{l}\text { No. leaves } \\
\text { plant }^{-1}\end{array}$ & $\begin{array}{c}\text { foliage } \\
\text { fresh } \\
\text { weight } \\
\text { (gm) }\end{array}$ \\
\hline \multirow{2}{*}{ Control } & $\mathrm{H} 0$ & & $59.4 \mathrm{ab}$ & $534.7 \mathrm{~g}$ & $31.8 \mathrm{~g}$ & $53.5 \mathrm{ab}$ & $425.0 \mathrm{e}$ & $25.2 \mathrm{e}$ \\
\hline & H1 & & $69.3 \mathrm{ab}$ & $856.8 \mathrm{e}$ & $36.3 \mathrm{ef}$ & $57.8 \mathrm{ab}$ & $692.0 \mathrm{~d}$ & $28.5 \mathrm{~d}$ \\
\hline \multirow[t]{2}{*}{25} & H0 & & $53.9 \mathrm{~b}$ & $699.5 \mathrm{f}$ & $35.9 \mathrm{f}$ & $55.2 \mathrm{ab}$ & $584.5 \mathrm{de}$ & $28.4 \mathrm{~d}$ \\
\hline & H1 & & $68.3 \mathrm{ab}$ & $1535.1 \mathrm{c}$ & $38.7 \mathrm{~cd}$ & $59.3 \mathrm{ab}$ & $1311.5 \mathrm{c}$ & $31.0 \mathrm{~b}$ \\
\hline \multirow[t]{2}{*}{50} & $\mathrm{H} 0$ & & $61.2 \mathrm{ab}$ & $1420.3 \mathrm{~d}$ & $37.1 \mathrm{e}$ & $53.0 \mathrm{ab}$ & $1194.0 \mathrm{c}$ & $29.5 \mathrm{~cd}$ \\
\hline & H1 & & $73.5 \mathrm{ab}$ & $1961.9 \mathrm{~b}$ & $39.7 \mathrm{~b}$ & $58.5 \mathrm{ab}$ & $1676.8 \mathrm{~b}$ & $31.5 \mathrm{~b}$ \\
\hline \multirow[t]{2}{*}{75} & Ho & & $59.5 \mathrm{ab}$ & $1539.5 \mathrm{c}$ & $38.3 \mathrm{~d}$ & $48.2 \mathrm{~b}$ & $1272.8 \mathrm{c}$ & $30.3 \mathrm{bc}$ \\
\hline & H1 & & $74.5 \mathrm{a}$ & $2997.8 \mathrm{a}$ & $44.1 \mathrm{a}$ & $64.8 \mathrm{ab}$ & $2491.8 \mathrm{a}$ & $35.2 \mathrm{a}$ \\
\hline \multirow[t]{2}{*}{100} & H0 & & $63.7 \mathrm{ab}$ & $1621.6 \mathrm{c}$ & $38.2 \mathrm{~d}$ & $55.2 \mathrm{ab}$ & $1343.2 \mathrm{c}$ & $30.3 \mathrm{bc}$ \\
\hline & H1 & & $74.7 \mathrm{a}$ & $3056.5 \mathrm{a}$ & $45.7 \mathrm{a}$ & $68.0 \mathrm{a}$ & $2573.0 \mathrm{a}$ & $35.5 \mathrm{a}$ \\
\hline \multirow[t]{2}{*}{ Control } & & Ch0 & $60.2 \mathrm{c}$ & $614.2 \mathrm{i}$ & $33.3 \mathrm{~h}$ & $53.0 \mathrm{a}$ & $498.7 \mathrm{i}$ & $26.5 \mathrm{~g}$ \\
\hline & & Ch1 & $68.3 \mathrm{a}-\mathrm{c}$ & $777.2 \mathrm{~h}$ & $34.8 \mathrm{~g}$ & $58.3 \mathrm{a}$ & $618.3 \mathrm{~h}$ & $27.2 \mathrm{f}$ \\
\hline \multirow[t]{2}{*}{25} & & Ch0 & $58.8 \mathrm{c}$ & $900.8 \mathrm{~g}$ & $35.9 \mathrm{f}$ & $55.8 \mathrm{a}$ & $782.2 \mathrm{~g}$ & $28.7 \mathrm{e}$ \\
\hline & & Ch1 & $63.5 \mathrm{bc}$ & $1333.8 \mathrm{f}$ & $38.8 \mathrm{~d}$ & $58.7 \mathrm{a}$ & $1113.8 \mathrm{f}$ & $30.7 \mathrm{c}$ \\
\hline \multirow[t]{2}{*}{50} & & Ch0 & $66.3 \mathrm{a}-\mathrm{c}$ & $1568.2 \mathrm{e}$ & $37.3 \mathrm{~g}$ & $52.2 \mathrm{a}$ & $1305.3 \mathrm{e}$ & $29.7 \mathrm{~d}$ \\
\hline & & Ch1 & $68.5 \mathrm{ab}$ & $1813.9 \mathrm{~d}$ & $39.6 \mathrm{c}$ & $59.3 \mathrm{a}$ & $1565.5 \mathrm{~d}$ & $31.2 \mathrm{bc}$ \\
\hline \multirow[t]{2}{*}{75} & & Ch0 & $59.7 \mathrm{c}$ & $2152.3 \mathrm{c}$ & $39.6 \mathrm{c}$ & $52.5 \mathrm{a}$ & $1770.5 \mathrm{c}$ & $31.5 \mathrm{bc}$ \\
\hline & & Ch1 & $74.3 \mathrm{ab}$ & $2384.9 \mathrm{a}$ & $42.7 \mathrm{a}$ & $60.5 \mathrm{a}$ & $1994.2 \mathrm{a}$ & $34.0 \mathrm{a}$ \\
\hline \multirow[t]{6}{*}{100} & & Ch0 & $61.6 \mathrm{c}$ & $2265.8 \mathrm{~b}$ & $41.0 \mathrm{~b}$ & $60.2 \mathrm{a}$ & $1893.3 \mathrm{~b}$ & $31.9 \mathrm{~b}$ \\
\hline & & Ch1 & $76.7 \mathrm{a}$ & $2412.3 \mathrm{a}$ & $43.0 \mathrm{a}$ & $63.0 \mathrm{a}$ & $2022.8 \mathrm{a}$ & $33.8 \mathrm{a}$ \\
\hline & $\mathrm{H} 0$ & Ch0 & $55.2 \mathrm{c}$ & $1054.9 \mathrm{~d}$ & $35.3 \mathrm{~d}$ & $50.9 \mathrm{c}$ & $871.1 \mathrm{~d}$ & $28.0 \mathrm{~d}$ \\
\hline & & Ch1 & $36.9 \mathrm{~b}$ & $1271.3 \mathrm{c}$ & $37.3 \mathrm{c}$ & $55.1 \mathrm{bc}$ & $1056.7 \mathrm{c}$ & $29.4 \mathrm{c}$ \\
\hline & H1 & $\mathrm{Ch} 0$ & $67.5 \mathrm{~b}$ & $1945.7 b$ & $39.5 \mathrm{~b}$ & $58.6 \mathrm{~b}$ & $1628.9 \mathrm{~b}$ & $31.3 \mathrm{~b}$ \\
\hline & & Ch1 & $76.6 \mathrm{a}$ & $2217.6 \mathrm{a}$ & $42.3 \mathrm{a}$ & $64.8 \mathrm{a}$ & $1869.2 \mathrm{a}$ & $33.4 \mathrm{a}$ \\
\hline
\end{tabular}

$* \mathrm{NK}(\%)=$ percentage of the recommended doses of NK fertilizers. $\mathrm{H} 0=$ control $\mathrm{H} 1=1000 \mathrm{ppm}$ humic acid $\mathrm{Ch} 0=$ control $\mathrm{Ch} 1=250 \mathrm{ppm}$ chitosan

*values followed by the same letter(s), within comparable group of means, do not significantly differ, using revised L.S.D. test at 0.05 level.

Table 5: Second order interaction effect among NK levels, humic acid and chitosan on vegetative characters of potato plants during the fall seasons of 2014 and 2015.

\begin{tabular}{|c|c|c|c|c|c|c|c|c|}
\hline \multicolumn{3}{|c|}{ Treatments } & \multicolumn{3}{|c|}{2014} & \multicolumn{3}{|c|}{2015} \\
\hline NK (\%) & $\begin{array}{c}\text { Humic } \\
\text { acid }\end{array}$ & Chitosan & $\begin{array}{l}\text { No. main } \\
\text { stems }^{\text {Plant }}{ }^{-1}\end{array}$ & $\begin{array}{l}\text { No. } \\
\text { leaves } \\
\text { Plant }^{-1}\end{array}$ & $\begin{array}{c}\text { foliage } \\
\text { Fresh } \\
\text { Weight } \\
\text { (gm) }\end{array}$ & $\begin{array}{l}\text { No. main } \\
\text { stems }_{\text {Plant }^{-1}}\end{array}$ & $\begin{array}{c}\text { No. } \\
\text { leaves } \\
\text { Plant }^{-1}\end{array}$ & $\begin{array}{c}\text { foliage } \\
\text { Fresh } \\
\text { Weight } \\
\text { (gm) }\end{array}$ \\
\hline \multirow[t]{4}{*}{ Control } & $\mathrm{H0}$ & Ch0 & $2.8 \mathrm{~d}$ & $29 \mathrm{hi}$ & $72.3 \mathrm{i}$ & $2.3 \mathrm{de}$ & $26.3 \mathrm{ef}$ & $61.3 \mathrm{~h}$ \\
\hline & & Ch1 & $3.2 \mathrm{~cd}$ & $42 \mathrm{c}-\mathrm{h}$ & $75.4 \mathrm{hi}$ & $2.7 \mathrm{cde}$ & $30.7 \mathrm{def}$ & $75.3 \mathrm{~h}$ \\
\hline & H1 & Ch0 & $3.6 \mathrm{~b}-\mathrm{d}$ & $47 b-f$ & $94.7 \mathrm{gh}$ & $3.0 \mathrm{bcd}$ & $34.3 \mathrm{c}-\mathrm{f}$ & $78.0 \mathrm{~h}$ \\
\hline & & Ch1 & $4.4 \mathrm{a}-\mathrm{c}$ & $49 \mathrm{~b}-\mathrm{d}$ & $99.0 \mathrm{~g}$ & $3.3 \mathrm{a}-\mathrm{d}$ & $37.7 \mathrm{bcd}$ & $92.0 \mathrm{fgh}$ \\
\hline \multirow[t]{4}{*}{25} & H0 & Ch0 & $2.8 \mathrm{~d}$ & $30 \mathrm{hi}$ & $101.7 \mathrm{fg}$ & $2.7 \mathrm{cde}$ & $29.7 \mathrm{def}$ & $92.0 \mathrm{fgh}$ \\
\hline & & Ch1 & $3.2 \mathrm{~cd}$ & $39 c-i$ & $100.9 \mathrm{~g}$ & $2.7 \mathrm{cde}$ & $28.7 \mathrm{def}$ & $87.3 \mathrm{gh}$ \\
\hline & H1 & Ch0 & $3.6 \mathrm{~b}-\mathrm{d}$ & $41 c-i$ & $110.2 \mathrm{e}-\mathrm{g}$ & $3.7 \mathrm{abc}$ & $40.0 \mathrm{abcd}$ & $95.3 \mathrm{fgh}$ \\
\hline & & Ch1 & $4.0 \mathrm{a}-\mathrm{d}$ & $51 \mathrm{bc}$ & $168.6 \mathrm{~d}$ & $3.7 \mathrm{abc}$ & $43.7 \mathrm{abc}$ & $144.3 \mathrm{de}$ \\
\hline \multirow[t]{4}{*}{50} & $\mathrm{HO}$ & Ch0 & $3.6 \mathrm{~b}-\mathrm{d}$ & $34 \mathrm{f}-\mathrm{i}$ & $123.3 \mathrm{ef}$ & $2.3 \mathrm{de}$ & $24.3 \mathrm{f}$ & $.114 .3 \mathrm{efg}$ \\
\hline & & Ch1 & $2.8 \mathrm{~d}$ & 36 e-i & $128.8 \mathrm{e}$ & $2.3 \mathrm{de}$ & $28.7 \mathrm{def}$ & 119.7 efg \\
\hline & H1 & Ch0 & $3.6 \mathrm{~b}-\mathrm{d}$ & $45 \mathrm{c}-\mathrm{g}$ & $158.9 \mathrm{~d}$ & 2.7 cde & $31.7 \mathrm{c}-\mathrm{f}$ & $137.7 \mathrm{de}$ \\
\hline & & Ch1 & $5.2 \mathrm{a}$ & $66 \mathrm{a}$ & $215.4 \mathrm{c}$ & $4.3 \mathrm{a}$ & $47.0 \mathrm{ab}$ & $184.7 \mathrm{~b}$ \\
\hline \multirow[t]{4}{*}{75} & H0 & Ch0 & $2.8 \mathrm{~d}$ & $38 \mathrm{~d}-\mathrm{i}$ & $152.7 \mathrm{~d}$ & $1.7 \mathrm{e}$ & $22.7 \mathrm{f}$ & $125.3 \mathrm{ef}$ \\
\hline & & Ch1 & $3.2 \mathrm{~cd}$ & $41 c-i$ & $170.1 \mathrm{~d}$ & $2.3 \mathrm{de}$ & $27.0 \mathrm{ef}$ & $149.3 \mathrm{cde}$ \\
\hline & H1 & Ch0 & $3.2 \mathrm{~cd}$ & $43 \mathrm{c}-\mathrm{g}$ & 206.9 c & $3.7 \mathrm{abc}$ & $37.0 \mathrm{~b}-\mathrm{e}$ & $179.7 \mathrm{bc}$ \\
\hline & & Ch1 & $4.8 \mathrm{ab}$ & $68 \mathrm{a}$ & $359.6 \mathrm{a}$ & $3.7 \mathrm{abc}$ & $50.7 \mathrm{a}$ & $304.0 \mathrm{a}$ \\
\hline \multirow[t]{4}{*}{100} & H0 & Ch0 & $2.8 \mathrm{~d}$ & $33 \mathrm{~g}-\mathrm{i}$ & $162.8 \mathrm{~d}$ & $3.0 \mathrm{bcd}$ & $32.7 \mathrm{c}-\mathrm{f}$ & $143.7 \mathrm{de}$ \\
\hline & & Ch1 & $4.0 \mathrm{a}-\mathrm{d}$ & $40 \mathrm{c}-\mathrm{i}$ & $173.2 \mathrm{~d}$ & $3.0 \mathrm{bcd}$ & $32.3 \mathrm{c}-\mathrm{f}$ & $165.7 \mathrm{bcd}$ \\
\hline & H1 & Ch0 & $4.8 \mathrm{ab}$ & $49 b-d$ & $208.8 \mathrm{c}$ & $4.0 \mathrm{ab}$ & $47.7 \mathrm{ab}$ & $180.7 \mathrm{bc}$ \\
\hline & & Ch1 & $4.8 \mathrm{ab}$ & $60 a b$ & $332.5 \mathrm{~b}$ & $4.3 \mathrm{a}$ & $50.3 \mathrm{a}$ & $284.7 \mathrm{a}$ \\
\hline
\end{tabular}

*NK $(\%)=$ percentage of the recommended doses of NK fertilizers. $\mathrm{H} 0=$ control $\mathrm{H} 1=1000 \mathrm{ppm}$ humic acid Ch0= control $\mathrm{Ch} 1=250 \mathrm{ppm}$ chitosan

*values followed by the same letter(s), within comparable group of means, do not significantly differ, using revised L.S.D.test at 0.05 level. 
Table 6: Second order interaction effect among NK levels, humic acid and chitosan on vegetative characters of potato plants during the fall seasons of 2014 and 2015

\begin{tabular}{|c|c|c|c|c|c|c|c|c|}
\hline \multicolumn{3}{|c|}{ Treatments } & \multicolumn{3}{|c|}{2014} & \multicolumn{3}{|c|}{2015} \\
\hline NK (\%) & $\begin{array}{c}\text { Humic } \\
\text { acid }\end{array}$ & Chitosan & $\begin{array}{c}\text { Plant } \\
\text { height } \\
(\mathrm{cm})\end{array}$ & $\begin{array}{l}\text { Leaf area } \\
\quad\left(\mathrm{cm}^{2}\right)\end{array}$ & $\begin{array}{c}\text { Leaf } \\
\text { chlorophyll } \\
\text { content } \\
(\mathrm{mg} / \mathbf{1 0 0 g} \mathrm{FW})\end{array}$ & $\begin{array}{c}\text { Plant } \\
\text { height } \\
(\mathrm{cm})\end{array}$ & $\begin{array}{l}\text { Leaf area } \\
\quad\left(\mathrm{cm}^{2}\right)\end{array}$ & $\begin{array}{c}\text { Leaf } \\
\text { chlorophyll } \\
\text { content } \\
(\mathbf{m g} / \mathbf{1 0 0 g} \\
\text { FW) }\end{array}$ \\
\hline \multirow[t]{4}{*}{ Control } & $\mathrm{HO}$ & Ch0 & $53.8 \mathrm{~cd}$ & $524.4 \mathrm{~m}$ & $31.4 \mathrm{~m}$ & $51.3 \mathrm{~cd}$ & $404.7 \mathrm{n}$ & $24.8 \mathrm{j}$ \\
\hline & & Ch1 & $64.9 \mathrm{a}-\mathrm{d}$ & $545.0 \mathrm{~m}$ & $32.3 \mathrm{~m}$ & $55.7 \mathrm{a}-\mathrm{d}$ & $445.3 n$ & $25.6 \mathrm{j}$ \\
\hline & H1 & $\mathrm{Ch} 0$ & $66.8 \mathrm{a}-\mathrm{c}$ & $704.1 \mathrm{kl}$ & $35.2 \mathrm{k}$ & $54.7 \mathrm{bcd}$ & $592.7 \mathrm{~lm}$ & $28.1 \mathrm{hi}$ \\
\hline & & Ch1 & $71.7 \mathrm{ab}$ & $1009.5 \mathrm{j}$ & $37.4 \mathrm{j}$ & $61.0 \mathrm{abc}$ & $791.3 \mathrm{k}$ & $28.9 \mathrm{gh}$ \\
\hline \multirow[t]{4}{*}{25} & $\mathrm{H} 0$ & Ch0 & $48.2 \mathrm{~d}$ & $593.4 \mathrm{~lm}$ & $34.1 \mathrm{i}$ & $56.3 \mathrm{a}-\mathrm{d}$ & $505.7 \mathrm{mn}$ & $27.0 \mathrm{i}$ \\
\hline & & Ch1 & $59.7 b-d$ & $805.6 \mathrm{k}$ & $37.8 \mathrm{ij}$ & $54.0 \mathrm{bcd}$ & 663.31 & $29.7 \mathrm{fg}$ \\
\hline & H1 & Ch0 & $69.4 \mathrm{a}-\mathrm{c}$ & $1208.2 \mathrm{i}$ & $37.7 \mathrm{ij}$ & $55.3 \mathrm{a}-\mathrm{d}$ & $1058.7 \mathrm{j}$ & $30.4 \mathrm{ef}$ \\
\hline & & Ch1 & $67.2 \mathrm{a}-\mathrm{c}$ & $1861.9 \mathrm{~d}$ & $39.8 \mathrm{e}$ & $63.3 \mathrm{abc}$ & $1564.3 \mathrm{~d}$ & $31.7 \mathrm{~cd}$ \\
\hline \multirow[t]{4}{*}{50} & $\mathrm{HO}$ & Ch0 & 65.3a-d & $1264.6 \mathrm{i}$ & $34.9 \mathrm{kl}$ & $50.7 \mathrm{~cd}$ & $1063.7 \mathrm{j}$ & $28.1 \mathrm{hi}$ \\
\hline & & Ch1 & $57.1 \mathrm{~b}-\mathrm{d}$ & $1575.9 \mathrm{fg}$ & $39.2 \mathrm{e}-\mathrm{g}$ & $55.3 \mathrm{a}-\mathrm{d}$ & $1324.3 \mathrm{gh}$ & 30.8 def \\
\hline & H1 & Ch0 & $67.2 \mathrm{a}-\mathrm{c}$ & $1871.9 \mathrm{~d}$ & $39.5 \mathrm{ef}$ & $53.7 \mathrm{bcd}$ & $1547.0 \mathrm{de}$ & $31.3 \mathrm{de}$ \\
\hline & & Ch1 & $79.9 \mathrm{a}$ & $2051.9 \mathrm{fg}$ & $39.9 \mathrm{ef}$ & $63.3 \mathrm{abc}$ & $1806.7 \mathrm{c}$ & $31.6 \mathrm{~cd}$ \\
\hline \multirow[t]{4}{*}{75} & $\mathrm{HO}$ & Ch0 & $52.6 \mathrm{~cd}$ & $1396.7 \mathrm{~h}$ & $37.7 \mathrm{ij}$ & $44.3 \mathrm{~d}$ & $1143.3 \mathrm{ij}$ & $30.1 \mathrm{f}$ \\
\hline & & Ch1 & $66.5 \mathrm{a}-\mathrm{c}$ & $1682.3 \mathrm{ef}$ & $38.8 \mathrm{f}-\mathrm{h}$ & $52.0 \mathrm{~cd}$ & $1402.3 \mathrm{fg}$ & $30.4 \mathrm{ef}$ \\
\hline & $\mathrm{H} 1$ & Ch0 & $66.8 \mathrm{a}-\mathrm{c}$ & $2907.9 \mathrm{~b}$ & $41.5 \mathrm{~d}$ & $60.7 \mathrm{abc}$ & $2397.7 \mathrm{~b}$ & $32.7 \mathrm{bc}$ \\
\hline & & Ch1 & $82.1 \mathrm{a}$ & $3087.7 \mathrm{a}$ & $46.7 \mathrm{~b}$ & $69.0 \mathrm{a}$ & $2586.0 \mathrm{a}$ & $37.6 \mathrm{a}$ \\
\hline \multirow[t]{4}{*}{100} & H0 & Ch0 & $56.0 \mathrm{~b}-\mathrm{c}$ & $1495.4 \mathrm{gh}$ & $38.1 \mathrm{ef}$ & $51.7 \mathrm{~cd}$ & $1238.3 \mathrm{hi}$ & $30.1 \mathrm{f}$ \\
\hline & & Ch1 & $71.3 \mathrm{ab}$ & $1747.7 \mathrm{de}$ & $38.3 \mathrm{~g}-\mathrm{i}$ & $58.7 \mathrm{abc}$ & $1448.0 \mathrm{ef}$ & $30.4 \mathrm{ef}$ \\
\hline & H1 & Ch0 & $67.2 \mathrm{a}-\mathrm{c}$ & $3036.1 \mathrm{a}$ & $43.8 \mathrm{c}$ & $68.7 \mathrm{a}$ & $2548.3 \mathrm{a}$ & $33.7 \mathrm{~b}$ \\
\hline & & Ch1 & $82.1 \mathrm{a}$ & $3076.9 \mathrm{a}$ & $47.6 \mathrm{a}$ & $67.3 \mathrm{ab}$ & $2597.7 \mathrm{a}$ & $37.2 \mathrm{a}$ \\
\hline
\end{tabular}

*NK $(\%)=$ percentage of the recommended doses of NK fertilizers. $\mathrm{H} 0=$ control $\mathrm{H} 1=1000 \mathrm{ppm}$ humic acid Ch0= control $\mathrm{Ch} 1=250 \mathrm{ppm}$ chitosan

* values followed by the same letter(s), within comparable group of means, do not significantly differ, using revised L.S.D.test at 0.05 level.

Ghasemi et al. (2012) reported that the highest leaf area, plant height, shoot dry weight, and number of leaves were found with the application of $200 \mathrm{~kg} / \mathrm{ha}$ of nitrogen fertilizer application treatments. Potassium promotes photosynthesis and transport assimilates of the carbohydrates to the storage organs. These results are in agreement with those of by Marschner (1986) and Sarrwy et al., (2010) who recorded that application of potassium, improved the chlorophyll. These results are in agreement with previous investigation illistrated by Zhang et al., (2002); Lin and Danfeng (2003). They found that increasing in vegetative growth, net photosynthetic rate; potassium content and chlorophyll content were associated with enhancement of potassium levels.

The present results of vegetative growth are in accordance to Abdel Fatah et al., (2008), who observed that application of humic acid improved growth parameters. The favorable effect of humic acid may be related to its action on increasing hormonal growth responses (Poapst et al., 1971; Vaughan, 1974).

The significant effect of chitosan on plant growth may be attributed to an increase in the enzyme activities of nitrogen metabolism (nitrate reductase, glutamine synthetase and protease) and increased photosynthesis which improve the plant growth (Gornik et al., 2008; Mondal et al., 2012). also, chitosan induce to synthesize plant hormones such as gibberellins. Furthermore, it enhances growth by some signaling pathways related to auxin biosynthesis via a tryptophan in dependent pathway (Uthairatanakij et al., 2007; El-Bassiony et al., 2014). Also, may be due to an increase in the availability and uptake of water and essential nutrients through adjusting cell osmotic pressure by increasing antioxidants and enzyme activities (Guan et al., 2009).

\section{Tubers yield and its components}

Data arranged in Tables 7 - 9 illustrated the main and different order interactions of the three studied factors on tubers yield and its components, in the two growing seasons.

Results in Table 7 show the main effects of the three studied factors. Increasing NK \% up to $100 \%$ of the recommended dose led to, progressive and significant, increases in tubers yield plant ${ }^{-1}$ or fed $\mathrm{f}^{-1}$ and weight of large and medium sized tubers plant ${ }^{-1}$. The highest two NK \% didn't, significantly, differ in their effect on tubers yield $\mathrm{fed}^{-1}$, in the $1^{\text {st }}$ season and tubers yield of both medium and small tubers size. 


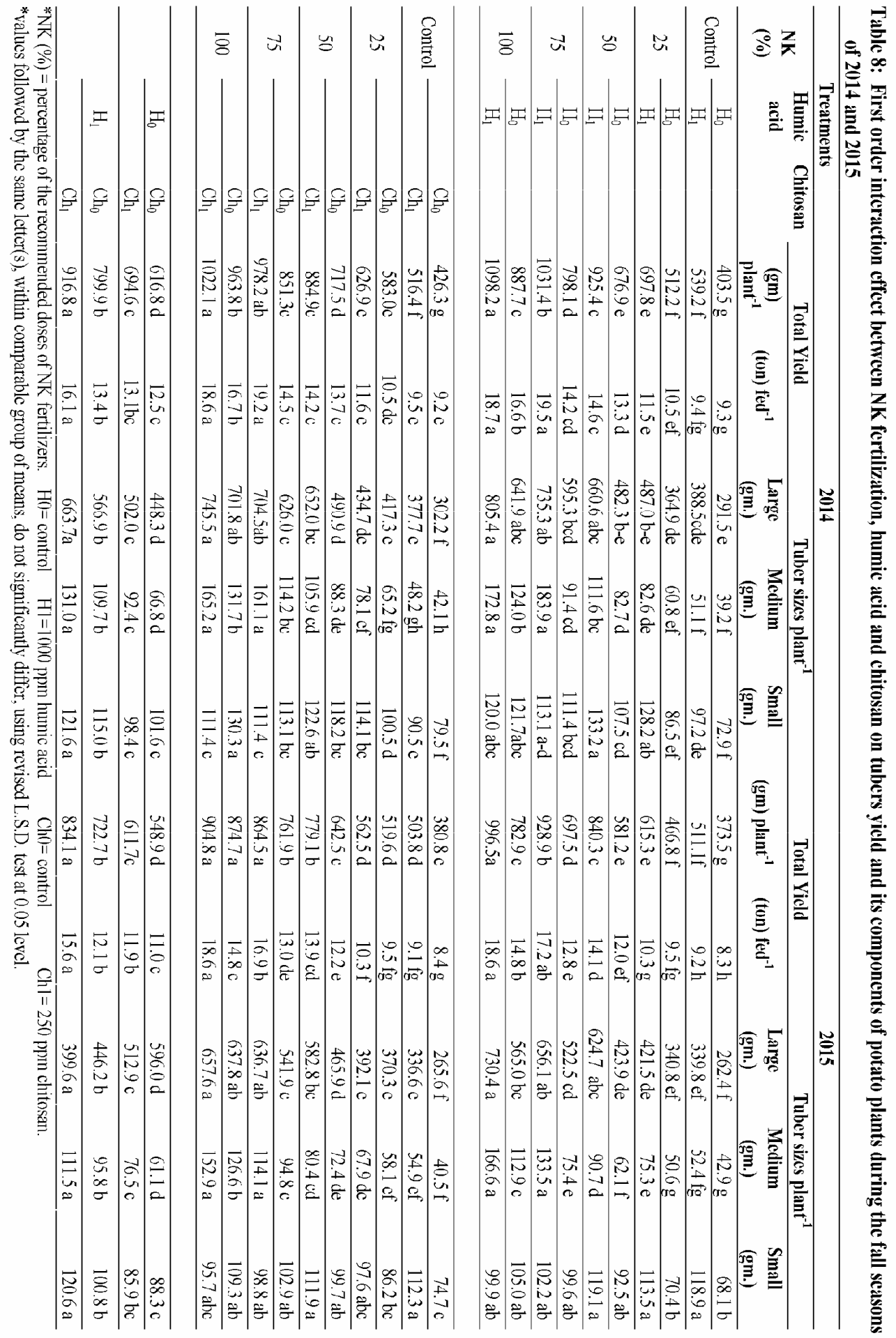




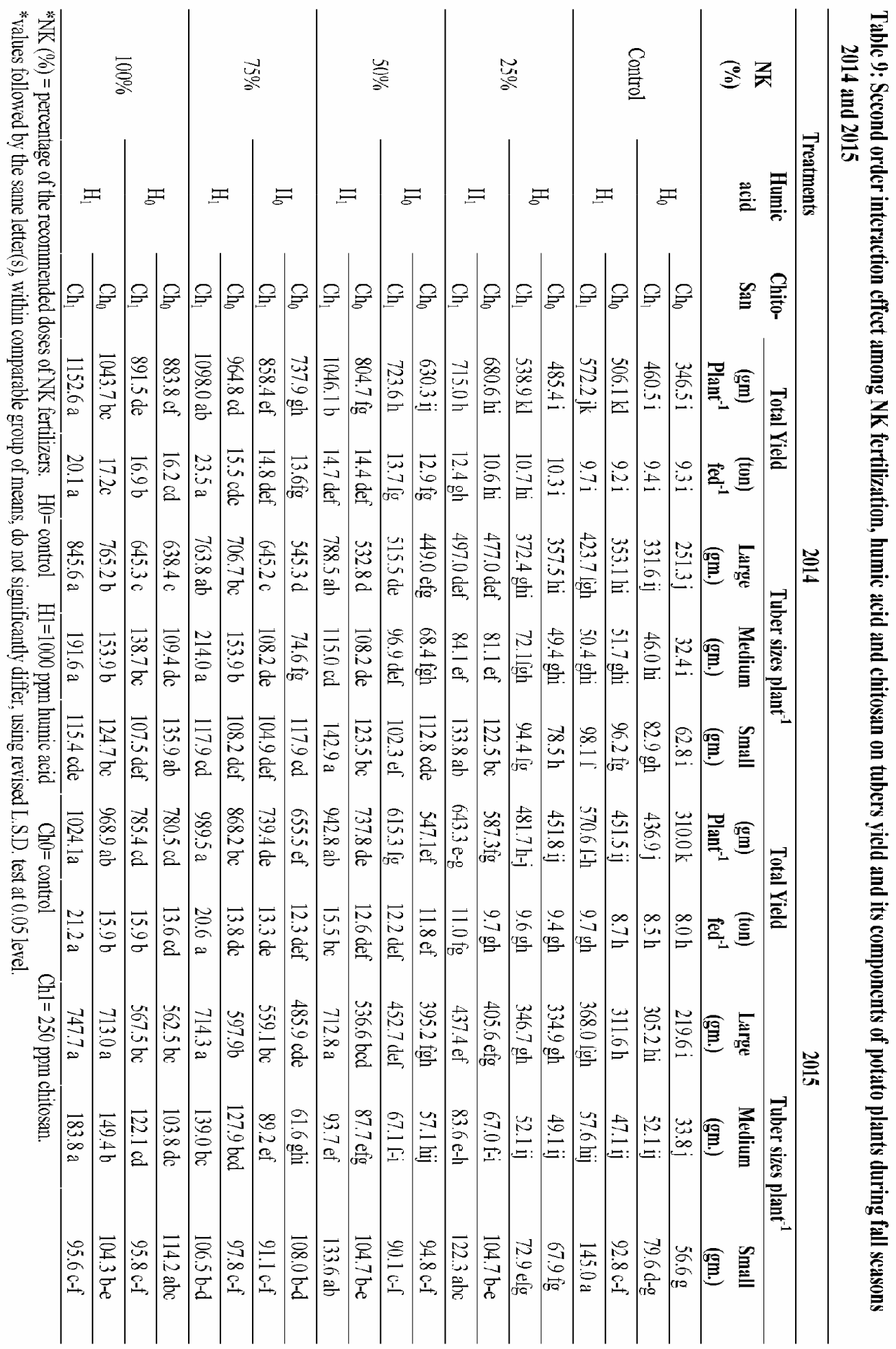


On the other hand, tubers yield of small sized plant ${ }^{-1}$ didn't, significantly, respond to different NK rates, in the $2^{\text {nd }}$ season. Humic acid application, significantly, produced higher tubers yield plant ${ }^{-1}$ or fed $^{-1}$ and three categories of tubers size than the untreated plants. Moreover, foliar application of chitosan, truly, increased tubers yield plant ${ }^{-1}$ or fed ${ }^{-1}$ and the heaviest weigh of medium sized tubers compared to control. The same trend was noticed concerning the yield of small size category in the first season. Meanwhile, the yield of large tubers didn't respond to chitosan treatments in the two seasons.

Table 8 reflects the effects of $1^{\text {st }}$ order interactions between any two studied factors on tubers yield and its components, in both seasons. Increasing NK applied rate up to $100 \%$ of the recommended dose combined with humic acid at $1000 \mathrm{ppm}$, significantly and constantly, increased tubers yield plant $^{-1}$ as compared to plants without treating of humic acid at any NK rate. Again, increasing NK applied level from 50 to $100 \%$ together with humic acid at $1000 \mathrm{ppm}$, truly, increased tubers yield $\mathrm{fed}^{-1}$ as compared to untreated plants of humic acid. Application of NK at $100 \%$ of the recommended dose together with humic acid at $1000 \mathrm{ppm}$ recorded the highest, intrinsic, mean value of large and medium sized tubers plant ${ }^{-1}$ whilst, the application of NK at 50\% combined humic acid at $1000 \mathrm{ppm}$ gained the highest small sized tubers plant ${ }^{1}$. The interaction between NK application at $100 \%$ and foliar spray of chitosan at $250 \mathrm{ppm}$ resulted, significantly, in the highest mean values of tubers yield plant ${ }^{-1}$ and $\mathrm{fed}^{-1}$, large and medium sized tubers plant $^{-1}$ meanwhile, the combination treatment of NK and chitosan at $0 \%$ and $0 \mathrm{ppm}$, respectively recoded the lowest mean value of small sized tubers plant ${ }^{-1}$, in both seasons with few exceptions. The effect of the interaction between humic acid and chitosan on tubers yield and its components was intrinsic. The highest mean magnitudes of tubers yield plant ${ }^{-1}$ and $\mathrm{fed}^{-1}$, categories of large, medium and small sized tubers plant $^{-1}$ were achieved due to the interactive treatment of humic acid at $1000 \mathrm{ppm}+$ chitosan at $250 \mathrm{ppm}$.

The impact of $2^{\text {nd }}$ order interaction among the three variables on tubers yield and its components was intrinsic, in both seasons (Table 9). Comparisons among the twenty mean values displayed that the growing potato plants fertilized with NK at 75 and/or $100 \%$ of the recommended NK rate and treated with humic acid at $1000 \mathrm{ppm}$ and foliar sprayed with chitosan at $250 \mathrm{ppm}$ recorded the highest, significant, mean value of tubers yield plant ${ }^{-1}$ and fed $^{-1}$, yield of large and medium sized tubers plant ${ }^{-1}$. Meanwhile, yield of small tubers plant ${ }^{-1}$ attained the highest mean value with the application of NK at $50 \%+$ humic acid at $1000 \mathrm{ppm}+$ chitosan at $250 \mathrm{ppm}$, in the $1^{\text {st }}$ season and $\mathrm{NK}$ at $0 \%$ + humic acid at $1000 \mathrm{ppm}+$ chitosan at $250 \mathrm{ppm}$, in the $2^{\text {nd }}$ season.
The beneficial application effect of NK at adequate rate on total tubers yield and its components could be related to the role of $\mathrm{N}$ in activating the vegetative growth parameters (Table 2 - 4). It's also, possible that sufficient quantity and perhaps the efficient absorption of $\mathrm{N}$ coupled together leads to more photosynthesis required for tubers formation. In addition, such increment in tuber yield may be due to the essential role of $\mathrm{K}$ in synthesis of carbohydrate necessary for formation and development of the tubers (Widdoson et al., 1974). Brouk (1985) reported that K application, positively affected the tubers yield of sweet potato via increasing the proportion of dry matter diverted from the foliage to the underground tuberous roots. The research results are agreement with many researchers who recorded an increase of potato tubers yield as a result of increasing the levels of potassium (K) fertilization. Such increases in tubers yield of potato was due to either formation of large size tubers or increasing of the number of tubers plant ${ }^{-}$ ${ }^{1}$ or both (El-Gamal, 1989). Several investigators came to similar conclusion (AL-Moshileh and Errebi, 2004; Misgina et al., 2016; Haddad et al., 2016).

Humic acid application was a good indicator of plant activation due to increased soil moisture content and nutrient availability, more number of stolons and taller length tuber ${ }^{-1}$ (Mahmoud and Hafez, 2010). Similar results were reported by Erik et al. (2000) and Samy et al. (2015). The increase in yield parameters might be due to beneficial effect of humic acid on plant foliage further than increase in tuber number /plant and tuber weight which reflect positively on increase of total yield. Humic acid act as bio stimulant induced hormonal activity of plant releasing different auxin types which in regulating plant growth Jensen (2004).

The enhancement effect of chitosan on yield potential might be due to chitosan have the simulative effect on physiological processes and improved the transportation of nitrogen in the functional leaves which improved vegetative growth and development (Chibu and Shibayama, 2003; Gornik et al., 2008). These results are agreeable with those recorded by Mondal et al. (2012) on okra, Shehata et al.(2012) on cucumber, Abu Muriefah (2013) on common bean and El-Miniawy et al. (2013)on strawberry.

\section{REFERENCES}

Ahmed, M. 2012. Effect of the application of humic substances on yield, quality and nutrient content of potato tubers in Egypt. Biochem. and Life Sci. pp 471-492.

Ahmad, A., M. Abd El-Baky, A. Ghoname, G. Riad and S. El-Abd. 2009. Potato tuber quality as affected by nitrogen form and rate. Mid. East. Russian J. Plant Sci. and Biotechno. 3: 47 - 52. 
Abdel Fatah, H., Gehan, Boshra, A., Shahin,S.M. (2008). The role of humic acid in reducing the harmful effect of irrigation with saline water on tifway turf. J. Bio. Chem. Environ. Sci., 3(1):7589.

Abu-Muriefah, S.Sharifa, 2013. Effect of chitosan on common bean (Phaseolus vulgaris L.) plants grown under water stress conditions. Inter. Res. J. Agric. Sci. and Soil Sci., 3(6):192-199.

Al-Moshileh A. M. and M. A. Errebi. 2004. Effect of various potassium sulfate rates on growth, yield and quality of potato grown under sandy soil and arid conditions. https://www.ipipotash.org/udocs/ Effect $\% 20$ of\%20Various\%20Potassium\%20Sulf ate $\% 20$ Rates $\% 20$ on.pdf

Al-Rawi, K, M. and A. M. Kalf-Allah. 1980. Design and analysis of agriculture experiments. Text book, El-Mosul Univ. Iraq. 487. p.

Black, C. A. 1965. Method of soil analysis. Amer. Soc. Agro. Nigh. Madison, Wis.

Bourke, R. M. 1985. Influence of nitrogen and potassium fertilizer on growth of sweet potato (Ipomoea batatas) in Papua New Guinea. Field Crops Res. 12: 363 - 375.

Bryan, H. and J. Stark. 2003. Humic acid effects on potato response to phosphorus. Idaho potato conference.http://www.extension.uidaho.edu/nutr ient/pdf/Potato/Humic\%20Acid\%20Effects\%20o n\%20Potato\%20REsponse \%20to\%20Phosphorus .pdf

Chibu, H., and H.Shibayama, 2003. Effects of chitosan application on the growth of several crops.In: Chitin and chitosan in life science. T. Uragami, K Kurita, and T. Fukamizo(eds.). Yamaguchi, Japan. pp.235-239.ISBN 4- 90646443-0.

El-Gamal, A. M. 1985. Effect of potassium level on potato yield and quality. J. Agric. Sci. Mansoura Univ. 10: 1473 - 1476.

El-Bassiony,A.M., Z.F.Fawzy, M.A. El-Nemr and Li Yunsheng, 2014. Improvement of growth,yield and quality of two varieties of kohlrabi plants as affected by application of some biostimulants. Middle East Journal of Agriculture Research 3(3): 491-498.

El-Miniawy,S.M., M.E.Ragab, S.M.Youssef and A.A. Metwally, 2013. Response of strawberry plants to foliar spraying of chitosan. Res. J. Agric. and Biol. Sci., 9(6): 366-372.

Erik, B., G. Feibert, C. C. Shock and L. D. Saundres. 2000. Evaluation of humic acid and other nonconventional fertilizer additives for onion productivity. Malheur Experiment Station, Oregon State University Ontario, Anabousi. http://www.cropinfo.net/pdf/ar/2000/2000-10OnionHumicAcid.pdf
Ghasemi, E., M. R. Tookaloo and H. R. Zabihi. 2012.. Effect of nitrogen, potassium and humic acid on vegetative growth, nitrogen and potassium uptake of potato mini-tubers in greenhouse conditions. Iranian J. Agro. and plant breeding, 8 (1): 39 - 56.

Gornik, K., M. Grzesik and B.R. Duda, 2008. The effect of chitosan on rooting of grape vine cuttings and on subsequent plant growth under drought and temperature stress. J. Fruit Ornamental Plant Res., 16: 333-343.

Guan, Y.J., J. Hu, X.J. Wang and C.X. Shao, 2009. Seed priming with chitosan improves maizegermination and seedling growth in relation to physiological changes.

Haddad, M., M. Nabeel Bani-Hani, J. A. Al-Tabbal and A. H. Al-Fraihat. 2016. Effect of different potassium nitrate levels on yield and quality of potato tubers. J. Food, Agric. and Enviro. 14 (1): $101-107$.

Halina K. S. Mazur (2008). Biological control of potato against Rhizoctonia solani. Scientific works of the Lithuanian institute of horticulture and Lithuanian university of agriculture. Sodininkyste IR darzininkyste, 27 (2): 419- 425.

Halina, K. S. Mazur (2008 a). The usefulness of chitosan and Pythium oligandrum in potato tuber protection against Helmintho -sporium solani. Folia Hort. Ann. 20(2): 67-74.

Halina, K. S. Mazur (2008 b). The effect of chitosan and Pythium oligandrum used in protection of potato tubers against late blight and soft rot. http://www.ptchit.lodz.pl/pliki/PTChit_\%28rw9b 4a3zik41webk\%29.pdf

Jensen, E. (2004) Seaweed; Fact or Fanc. From the Organic Broadcaster,Published by Moses the Midwest Organic and Sustainable Education. From the Broad Caster. 12(3): 164-170.

Krishnappa, K. S. 1989. Effect of fertilizer applications on dry matter and $\mathrm{N}, \mathrm{P}$ and $\mathrm{K}$ accumulation in the potato at different stages of growth. Mysore J. Agric. Sci. 23: 349 -54.

Lin Duo and Danfeng, H. (2003). Effects of potassium levels on photosynthesis and fruit quality of muskmelon in culture medium. Acta Horticulturae Sinica., 30(2): 221-223.

Mahmoud, A. R. and M. H. Magda. 2010. Increasing productivity of potato plants (Solanum tuberosum L.) by using potassium fertilizer and humic acid application. Inter. J. Acad. Res. 2: 83 -88 .

Marschner, H. (1986). The Mineral Nutrition of Higher Plants. 1st Ed. Academic Press, New York.Mohsen Kazemi BEPLS Vol 2 [11] October 20136 | P a g e C2013 AE LS, India 
Misgina, N. A. 2016. Effect of phosphorus and potassium fertilizer rates on yield and yield components of potato (Solanum tuberosum L.) at K/A wlaelo, Tigray, Ethiopia. Food Sci. Quality Manage. 48: 60 - 69.

Mengel, K. and E.A. Kirkby. 1987. Principle of plant nutrition. $4^{\text {th }} \mathrm{Ed}$. International Potash Institute. Pern. Switzerland. PP. 687.

Mohamed, E., I. Badawy and E. I. Rabea. 2011. A Biopolymer Chitosan and its derivatives as promising antimicrobial agents against plant pathogens and their applications in crop protection. Inter. J. Carbohydrate Chem. 2011, 460381, $29 \mathrm{p}$.

Mondal, M.M.A., M.A.Malek, A.B.Puteh, M.R.Ismail, M. Ashrafuzzaman and L. Naher, 2012. Effect of foliar application of chitosan on growth and yield in okra. A.J.C.S., 6: 918-921.

Pervez, M. A., C. Ayyub, M., M. R. Shabeen and M. A. Noor. 2013. Determination of physio morphological characteristics of potato crop regulated by potassium management. Pakistan J. Agric. Sci. 50: 611 - 615.

Poapst, P.A. and M. Schnitzer.1971. Fulvic acid and adventitious root formation. Soil boil. And Biochem. 3: 215-219.

Samy, M. M., N. A. Mohamed and M. G. Abd ElAziz. 2015. Effect of boron, copper and humic acid treatments on vegetative growth, yield and storability of Jerusalem artichoke tubers. J. Product. Dev. 20 (3): 325 - 342.
Sarrwy, S.M.A., Enas A., Hassan, H.S.A. (2010). Effect of foliar spray with potassium nitrate and mono-potassium phosphate on leaf mineral contents, fruit set, yield and fruit quality of Picual olive trees grown under sandy soil conditions. American-Eurasian J. Agric and Environ. Sci., 8(4): 420-430.

Selim, E. M., A. S. El-Neklawy and S. M. El-Ashry. 2009. Beneficial effects of humic substances fertigation on soil fertility to potato grown on sandy soil. Aust. J. Basic and Appl. Sci. (4): 4351- 4358.

Sheikha, S. A. K. and F. M. AL-Malki. 2011. Growth and chlorophyll responses of bean plants to the chitosan applications. Euro. J. Scienti. Res. 50 (1): $124-134$.

Uthairatanakij. A., Jaime, A.T.Silva and K.Obsuwan, 2007. Chitosan for improving orchidproduction and quality. Orchid Science and Biotechnology. Global Science Books

Vaughan, D.1974. A possible mechanism for humic acid action on cell elongation in root segments of Possum sativum under aspectic conditions. Soil Biol. Bio-chem. 6: 241-247.

Widdoson, F. V., A. Penny and R. C. Flint. 1974. Results from experiments measuring the effect of large amount of fertilizer and farmyard manure on main-crop potatoes grown in sandy soil at Woburn, Bedfordshire. J. Agric. Sci. Camb. 82: $117-127$.

Zhang, A., Huang Dan, F., Hou, Z. (2002). Effect of potassium nutrient on development and photosynthesis of melon plant. Journal of Shanghai Agricultural College, 20 (1): 13-17. 


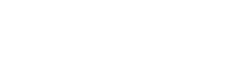

\section{تأثيرال حمض الهيوميك والكيتوزلن تهت طستوبلت مختلفة من النسميد النيتروجيفي والبوتلبي عل النوو وحصط المرنات ومكونالته في البلملس}

\section{إيملن عبد اللل حرفوش، علي هسن عبد الرازق، فتهي البرالهيم الأدغم ومحمد عبد العزيز المشرقاوي}

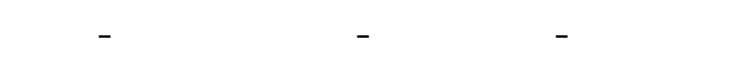

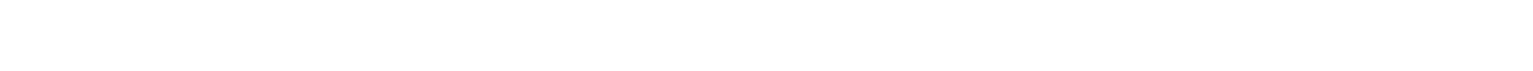

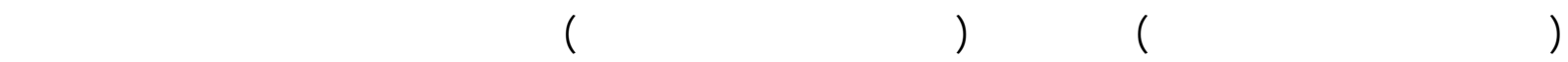

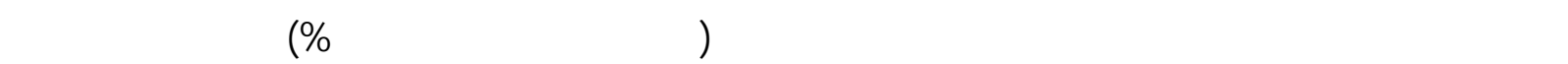

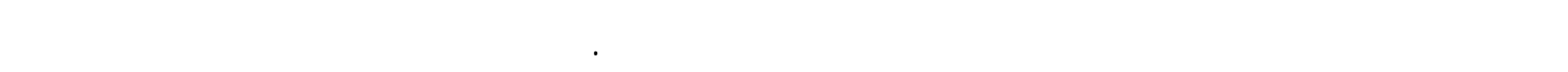

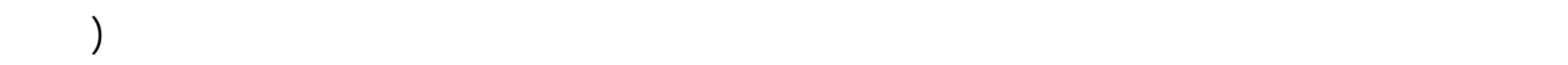

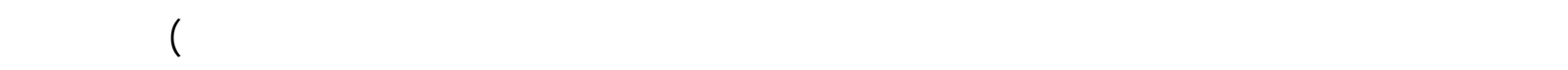

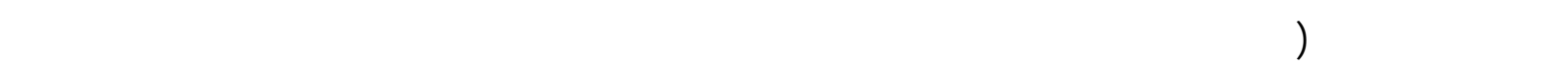

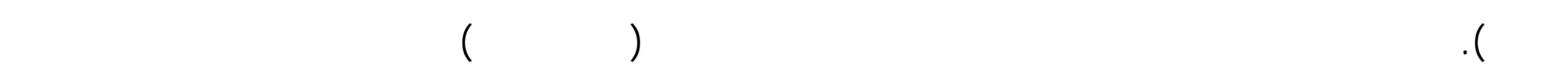

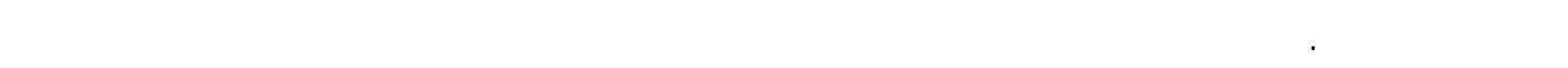

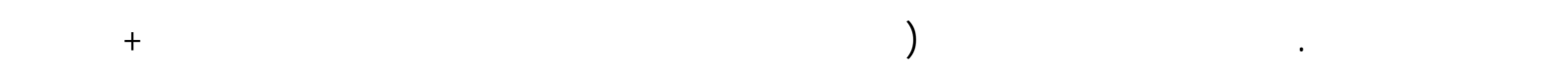

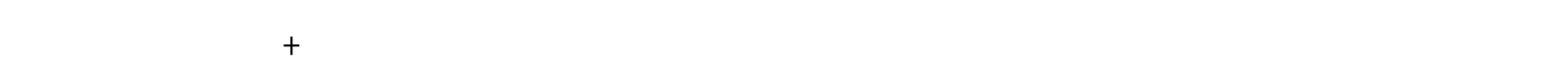

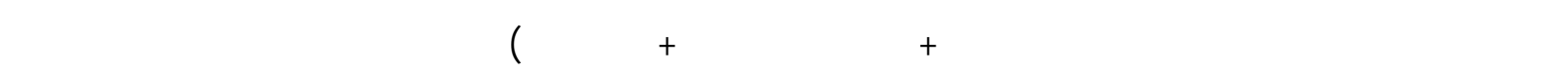

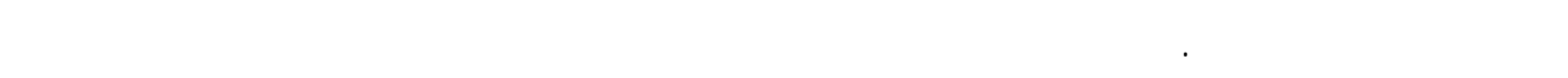

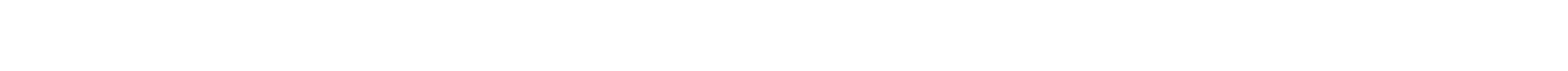
والكيتوزان وذك للحصول على أفضل النتائج. 